\title{
Joint Radio Resource Management based on the Species Competition Model
}

\author{
Yang Guang ${ }^{1}$, Chen $\mathrm{Jie}^{1}$, Yu Kai ${ }^{1}$, Zhang Ping ${ }^{1}$, Victor O.K. $\mathrm{Li}^{2}$ \\ (1. WTI Labs, Beijing University of Posts and Telecommunications, Beijing 100876, China; \\ 2. Multimedia Networking Lab, Department of Electrical and Electronic Engineering, University of \\ Hong Kong, Hong Kong, China ) \\ yanggguang@vip.sina.com
}

\begin{abstract}
For optimal radio resource utilization in heterogeneous wireless networks, Joint Radio Resource Management (JRRM) is required. In distributed JRRM, each radio each access network (RAN) adjusts network parameters to affect user's RAN selection, thereby indirectly implementing joint radio resource allocation. The mathematical method for instructing such adjustment is lacking. In this article, the relationship between different RANs is mapped into the competition between species in the well-known $L-V$ model developed by ecologists. Based on this model, an adjustment algorithm of distributed joint radio resource allocation is proposed. The simulation results show that compared with no adjustment or over adjustment, our adjustment algorithm can: 1) obtain proper resource allocation; 2) guarantee network coexistence.
\end{abstract}

Index Terms-Heterogeneous wireless network, joint radio resource management, species, $\mathrm{L}-\mathrm{V}$ model

\section{INTRODUCTION}

A Beyond $3 \mathrm{G}$ system is a heterogeneous network [1], where the multiplicity of radio access technologies as well as the diversity of terminals with reconfigurable capabilities will allow users to access different radio networks and to dynamically utilize different radio resources. Therefore, fueled by the increasing demands on global efficiency of radio resources, it is necessary to perform Joint Radio Resource Management (JRRM), which leads to optimal resource allocation for mobile subscribers.

Existing JRRM schemes can be classified into two categories: centralized JRRM [2-5] and distributed JRRM [6] [7]. Centralized JRRM, based on the tight-coupling architecture, collects all the related information (e.g. network status, user behavior, traffic volume, etc.) and executes certain algorithms to achieve the global optimal resource allocation. Distributed JRRM, in contrast, is based on the loose-coupling architecture, where each node in the system merely attempts to perform local resource allocation. Compared with centralized JRRM, distributed JRRM is less complex and more flexible. However, the resource allocation obtained is not a global optimum in most cases [6] [7].

In distributed JRRM, the exchange of information with the neighboring network is of great importance in evaluating the feasibility of resource allocation. The locally optimal resource allocation of one radio access network (RAN) may cause negative effects on neighboring RAN. The above problem has attracted attention in related search area, and an interactive procedure between local RAN and neighboring RAN is required to guarantee the allocation schemes suitable for all the involved RANs. However, no mathematical theory is available to instruct the distributed joint radio resource management in a quantitative manner.

The contribution of this article falls is in mathematical modeling for distributed joint radio resource management. Through mapping the relationships between different RANs in the heterogeneous system into the competitive relationships between different species, we propose a quantitative adjustment algorithm of distributed joint radio resource allocation based on the L-V model of species competition in ecology.

In Section II, we present a distributed joint radio resource management mechanism in which distributed control entities adjust network parameters to affect user's selection of RAN instead of directly allocating radio resources to users. Next, the basic concept and mathematical function of the L-V model are given in Section III. Subsequently, the mapping of heterogeneous wireless network to the L-V model is described in Section IV, and the mathematical representation of the competitive relationship between distributed RANs is defined. An adjustment algorithm of distributed joint radio resource allocation based on the L-V model is introduced in Section V. In Section VI, the simulation results show that two RANs can coexist and both can achieve certain profits by quantitative adjustments. Finally, summarizing remarks are provided. 


\section{Distributed Joint Radio Resource Management}

\section{A. Overview}

In this article, we propose a novel mechanism for distributed joint radio resource management. This mechanism is applied to the heterogeneous system composed of two different RANs covering a common area. Each RAN adopts specific radio access technology (RAT), leading to distinction in coverage range and bandwidth. The distributed JRRM entities, deployed in each RAN, maintain logical connections and exchange necessary information with each other. The users can select any of the RANs in this heterogeneous environment via reconfigurable terminals.

In our distributed JRRM mode, each RAN does not perform radio resource allocation directly. Instead, the RAN adjusts the network parameters to achieve the predefined goals according to the network status. Upon finishing the adjustment, such network parameters are broadcasted to the users to assist them in network selection. Therefore, the control entities obtain distributed joint radio resource allocation by network parameter adjustment. It should be noted that the above procedures are implemented by the cooperation between user terminal, radio network access module, radio network management module, and neighboring radio network management module. Specifically, taking the heterogeneous wireless network comprising radio networks 1 and 2 for instance, the adjustment

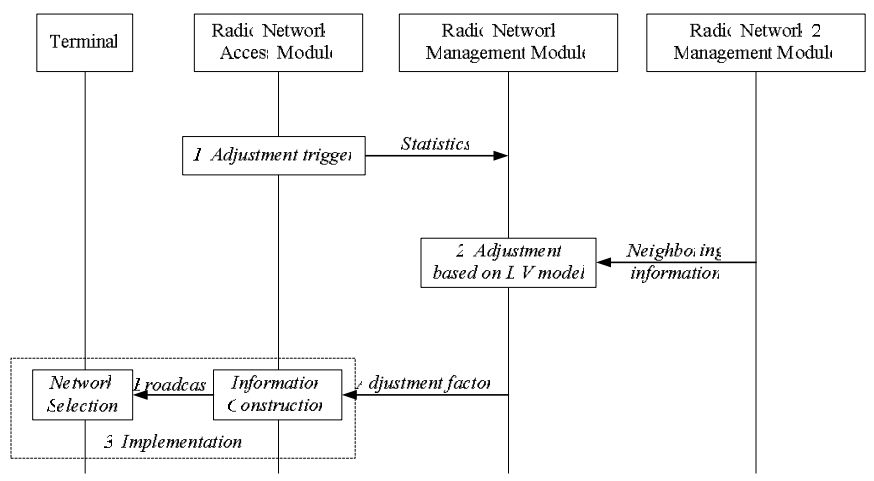

Fig. 1. Adjustment process of distributed joint radio resource management.

procedure of radio network 1 covers the following three steps as depicted in Fig.1. (Radio network 2 performs a similar procedure, which is not shown).

Step 1 Adjustment trigger: access module monitors the radio network statistics (e.g. traffic, the call dropping rate, delay etc.) in real-time. If some unexpected situations are detected, e.g. very high (low) traffic volume, high dropping rate, considerable delay etc. the adjustment is triggered and such statistics are submitted to the management module.

Step 2 Adjustment: once the adjustment is triggered, the management module is responsible for adjusting the network parameters. Note that the network parameters addressed here are information used by users when they select the access network. In this paper, the network parameters are defined as adjustment factors, involving price $(P)$ and network support bandwidth $(B)$. The management module, according to different trigger situation, uses predefined adjustment algorithm to obtain the adjustment results.

Step 3 Implementation: the management module feeds the adjustment factors back to this access module. The latter broadcasts such information to all users within the coverage area. Upon receiving the information, the user will select the RAN based on the price and network support bandwidth.

\section{B. Related Problem}

With respect to the aforementioned distributed JRRM, there are some issues. After adjustment is triggered, the management module will calculate the adjustment results. Firstly, the management module should determine the adjustment direction. For example, if network traffic load is high, or negotiated QoS level is unsatisfied, the management module should adjust the network parameters to raise the price or lower the network support bandwidth to discourage users from accessing this network. On the contrary, when the network traffic load is light, indicating that the resource is not fully exploited, the management module should adjust the network parameters to reduce the price or increase the network support bandwidth to attract more users to access this network. Secondly, the adjustment range should be quantified after the adjustment direction is determined. A low adjustment amplitude may not achieve the expected objective; while, an adjustment amplitude what is too high may cause users to migrate from one RAN to another, leading to overloading of the latter and possible oscillating migration over the two RANs Consequently, an algorithm to determine the adjustment range should be designed accounting for the objective of the local network and the possible effect on the neighboring network.

\section{L-V MODEL}

As mentioned above, there is no mathematical method to instruct the adjustment of the distributed JRRM currently. In this article, we find that the relationship between heterogeneous wireless networks in distributed JRRM is similar to the relationship between species in ecology; therefore the mathematical method in ecology can be adapted to instruct the above adjustment. The basic concept of $\mathrm{L}-\mathrm{V}$ model is presented next and the mapping of heterogeneous wireless network into $\mathrm{L}-\mathrm{V}$ model will be described in Section IV.

In ecology, a species can be defined as one group of life-form that occupies the same space, time and resource as another species. Two species can affect each other or be irrelevant, i.e. the relationship between different species can be diverse. However, the competitive relationship is the most common where the conflict of resource usage by inter-species, competition leads to variance of species numbers.

Lotka and Votterra have proposed a set of equations to represent the number variation caused by species competition as follows: 


$$
\begin{aligned}
& \frac{d N_{1}}{d t}=r_{1} N_{1}\left(\frac{K_{1}-N_{1}-\alpha_{12} N_{2}}{K_{1}}\right) \\
& \frac{d N_{2}}{d t}=r_{2} N_{2}\left(\frac{K_{2}-N_{2}-\alpha_{21} N_{1}}{K_{2}}\right)
\end{aligned}
$$

This mathematical model is called the $\mathrm{L}-\mathrm{V}$ model, where $N_{i}$ denotes the number of species $i, K_{i}$ denotes the environment load of species $i, r_{i}$ denotes the transient rising rate of species $i$ and $\alpha_{i j}$ denotes the competition coefficient. $K_{i}$ and $\alpha_{i j}$ can be interpreted as follows. $K_{i}$ represents the amount of species $i$ which the current environment can support when there is no competition. The detailed effect of $\alpha_{i j}$ is explored here: $\alpha_{12}$ is the competition coefficient of species 2 to species 1 , representing the restraining influence on species 1 by an individual of species 2. The effect of $\alpha_{21}$ is similar to $\alpha_{12}$. From the competition coefficient, we can deduce which species is restrained, which species performs a restraining influence and the amount of the restraining effect. For example, if $\alpha_{12}=0.5$, then the individual utilization of resource by species 2 corresponds to half of that in species 1 .

The different combinations of $K_{1}, K_{2}, \alpha_{12}$ and $\alpha_{21}$ give four possible cases: (a) $K_{l} / \alpha_{12}<K_{2}$ and $K_{2} / \alpha_{21}<K_{1}$; (b) $K_{1} / \alpha_{12}<$ $K_{2}$ and $K_{2} / \alpha_{21}>K_{l}$; (c) $K_{1} / \alpha_{12}>K_{2}$ and $K_{2} / \alpha_{21}<K_{l}$; (d) $K$ ${ }_{1} / \alpha_{12}>K_{2}$ and $K_{2} / \alpha_{21}>K_{l}$. According to the L-V model, species can coexist and keep equilibrium only in Case d. Here, coexistence and equilibrium mean that no species will die out. Therefore, equilibrium is satisfied when:

$$
\begin{aligned}
& K_{1 /} \alpha_{12}>K_{2} \\
& \text { and } K_{2 /} \alpha_{21}>K_{1}
\end{aligned}
$$

In equilibrium, $\frac{d N_{1}}{d t}=\frac{d N_{2}}{d t}=0$; hence from (1) and (2), we have

$$
\begin{aligned}
& \frac{K_{1}-N_{1}-\alpha_{12} N_{2}}{K_{1}}=0 \text { or } N_{1}=K_{1}-\alpha_{12} N_{2} \\
& \frac{K_{2}-N_{2}-\alpha_{21} N_{1}}{K_{2}}=0 \text { or } N_{2}=K_{2}-\alpha_{21} N_{1}
\end{aligned}
$$

Based on above theory, species amount can be determined and corresponding adjustment can be executed by utilizing inequalities (3) and (4). Furthermore, we can estimate species amount in equilibrium according to equations (5), (6). Hence, if the relationship among heterogeneous network can be mapped into species competitive relationship, the above mathematical can be adapted to instruct adjustment of distributed joint radio resource allocation and evaluate adjustment results.

\section{Mapping OF Heterogeneous Wireless Network INTO L-V MODEL}

\section{A. Mapping}

Different RANs in heterogeneous system offer communication services to users within the co-coverage area, and a user selects one RAN to obtain services. RANs attract users with low price and high bandwidth in order to achieve more operational profit. Users can be mapped into the resource, services in different wireless networks can be mapped into different species and user attraction to different RANs can be mapped into species competition due to the limited user number within the co-coverage area. When the service starts, one RAN occupies the user until the service ends; therefore the start and the end of services can be mapped into the birth and death of species.

As a result, we can apply the relationship of different species to reflect the relationship of different RANs in the distributed JRRM. Different RANs can compete with each other freely under the distributed JRRM's control, and the goal of the distributed JRRM is for all RANs to obtain operational profit without any RAN being ruined. Therefore, equilibrium in ecology can be mapped into profit balance in JRRM.

Furthermore, according to the environment load definition in ecology given in section III, the environmental load in JRRM can be defined as traffic possibly generated by all users within the network's coverage areas. The environment load changes when user number within the coverage or communication link quality varies. The mapping of heterogeneous wireless network into the $\mathrm{L}-\mathrm{V}$ model is shown in Table $\mathrm{I}$.

$$
\text { TABLE I }
$$

MAPPING BETWEEN THE L-V MODEL AND HETEROGENEOUS WIRELESS NETWORK

\begin{tabular}{ll}
\hline \multicolumn{1}{c}{ L-V model } & \multicolumn{1}{c}{ NETWORK } \\
\hline Different species & User services of different RANs \\
Species amount & Traffic in different RANs \\
Resource & User within network coverage area \\
Death & Service end \\
Birth & Service start \\
Equilibrium & Profit balance \\
Environmental load & Traffic supported by current users within network
\end{tabular}

\section{B. Competition Coefficient}

According to above mapping relationship, we can define competition coefficients between different RANs in JRRM. In heterogeneous network involving RAN 1 and 2, there is a pair of competition coefficients $\alpha_{21}$ and $\alpha_{12}$.

Competition coefficients reflect the competitive relation between different RANs and can be associated with price and network support bandwidth of different RANs. In this context, we define the variable affecting competition coefficients as follows:

Price: $P \in\left[P_{\min }, P_{\max }\right]$, where $P_{\min }$ is the minimum price required by network and $P_{\max }$ is the maximum price bearable to users; 
Network support bandwidth: $B \in\left[B_{\min }, B_{\max }\right]$, where $B_{\max }$ is the maximum bandwidth supported by RANs and $B_{\min }$ is the minimum bandwidth required for user services.

Based on above variable definition, the competition coefficients can be formulated as follows, in which $B_{i}$ and $P_{i}$ are the network support bandwidth and price of network $\mathrm{i}, \mathrm{i}=1,2$.

$$
\begin{aligned}
& \alpha_{21}=\frac{P_{2} / P_{1}+k B_{1} / B_{2}}{\beta} \\
& \alpha_{12}=\frac{P_{1} / P_{2}+k B_{2} / B_{1}}{\beta}
\end{aligned}
$$

Taking the competition coefficient $\alpha_{21}$ for instance, we can explain the notion above as follows:

Price factor $P_{2} / P_{1}$ reflects the competition degree of network 1 over network 2 on price;

Bandwidth factor $B_{1} / B_{2}$ reflects the competition degree of network 1 over network 2 on network support bandwidth;

Weighted factor $k$ reflects the relative importance of price and bandwidth to the users. If users consider price more important than network support bandwidth, then $\mathrm{k}<1$; otherwise, $\mathrm{k}>1$.

$\beta$ normalizes the value of the competition coefficients into $[0,1]$. It represents the possible maximal difference between price and network support bandwidth in the heterogeneous wireless network and can be formulated as follows:

$$
\beta=P_{\max } / P_{\min }+k B_{\max } / B_{\min }
$$

\section{Adjustment Algorithm}

Based on the above mapping relationship and the definition of competition coefficient, we present a novel algorithm for network parameter adjustment based on the L-V model. The details of this algorithm are described as follows.

\section{A. Adjustment Trigger}

The normal operating condition of two networks which do not require network parameter adjustment is considered to be equilibrium. In other words, if the relationship between environment load and competition coefficient meets the requirements of inequalities (3) and (4), the two RANs can coexist and maintain equilibrium. Otherwise, the radio network statistics (e.g. traffic, call dropping rate and delay etc.) exceeds a threshold, and the equilibrium is considered broken.

When the networks operate normally without adjustment, the price and the network support bandwidth will not change. Consequently, the competition coefficients of the two networks will not change either. At this time, only the change of the environmental load can cause the violation of inequalities (3) and (4).

The variance of the environmental load mainly depends on the number of users in the coverage area and the communication quality. If the number of users or the communication quality changes sharply, the environmental load will change dramatically and the equilibrium may be broken, triggering the adjustment of network parameters.

\section{B. Adjustment}

Once the adjustment has been triggered, based on the L-V model, the adjustment direction can be determined by monitoring the change of the environmental load. Take network 1 as an example, when $K_{l}$ decreases below a threshold so that the equilibrium is broken, since the constraint (3) is broken while (4) still holds to restore the equilibrium, the competition coefficient $\alpha_{12}$ must be reduced. Therefore, the network 1 parameter adjustment direction is determined.

After that, the adjustment range needs to be determined according to the following two constraints:

Firstly, $\alpha_{12}$ should be decreased enough to satisfy constraint (3);

Secondly, based on the definition of competition coefficient, while adjusting network 1 price and bandwidth to reduce $\alpha_{12}$, the competition coefficient $\alpha_{21}$ will correspondingly increase.

The increase of $\alpha_{21}$ is constrained by (4), i.e. the impact introduced by network 1 adjustment should not break the other equilibrium constraint.

Based on the above, we propose the following adjustment algorithm:

Step 1. Determining network 1 adjustment direction, which is to decrease $P_{l}$ and increase $B_{l}$ in this example;

Step 2. Under current $B_{1}$, Competition coefficients are functions of $P_{1}\left(P_{2}\right.$ and $B_{2}$ can be acquired from the neighboring network). Through inequalities (3) and (4), the adjustable lower bound $P_{\min A}$ and upper bound $P_{\max A}$ of $P_{I}$ can be obtained. From equations (7), (8) and constraint (3), (4), $P_{\min \_A}$ and $P_{\max \_A}$ can be expressed as follows:

$$
\begin{aligned}
& P_{l}<P_{2} /\left(\beta K_{1} / K_{2}-k B_{1} / B_{2}\right)=P_{\text {max }_{-} A} \\
& P_{l}>P_{2} \times\left(\beta K_{2} / K_{l}-k B_{2} / B_{I}\right)=P_{\text {min_A }} A
\end{aligned}
$$

Step 3. If and only if $P_{\max _{-} A}>P_{\min \_}, P_{\max A}>P_{\min }$ and $P_{\min \_}<P_{\max }$, go to Step 4; otherwise, increase $B_{1}$. If the current $B_{I}$ is greater than $B_{\max }$, go to step 5; otherwise go to Step 2.

Step 4. Randomly select a value from the range $\left[P_{\min \_A}, P_{\max \_} A\right]$ as the price $\mathrm{P}_{1}$ 's adjustment result. Now, the adjustment process ends with the current value of $B_{l}$ and $P_{l}$.

Step 5. $B_{l}$ has exceeded network support bandwidth $B_{\max }$, and no appropriate adjustment can meet constraint (3). Hence, error condition is reported to upper layer management module to indicate that there is no adjustable result available in such situation.

\section{Species Amount Estimation}

After the adjustment process, we can use equations (5) and (6) to estimate the species amount, i.e. traffic, of the two networks at the new equilibrium point. According to the L-V model equilibrium constraint, the above algorithm can control 
the traffic of different networks and ensure that they vary within a certain range, and as a consequence, neither of the network traffic would diminish. On the contrary, if there is no proper adjustment when the balance is broken, one of the two networks will die out (i.e. zero traffic).

\section{Simulation Results}

In the simulation we assume that the heterogeneous wireless network is comprised of RAN 1 and 2. At the beginning of the simulation, the two RANs have the same environmental load, network support bandwidth and price. Therefore, the initial competition coefficients $\alpha_{12}$ and $\alpha_{21}$ are equal, the initial traffic' $N_{1}$ and $N_{2}$ are equal as well. We also assume that: network support bandwidths are discrete values within a certain range, prices are continuous values within another range and the environmental loads $K_{1}$ and $K_{2}$ change at random.

This simulation compares three adjustment cases: No adjustment (No Ad); over adjustment (Over Ad), i.e., the maximal adjustment amplitude will be performed whenever adjustment is triggered; adjustment based on the L-V model, in which our adjustment algorithm is adopted. We observe the following statistics in the simulation: environmental load (EL), adjustment factors (AF), competition coefficient (CC) and traffic (TR).

\section{A. No Adjustment or Over Adjustment}

If there is no adjustment or over adjustment, the variation of the traffic of the two RANs $N_{1}$ and $N_{2}$ with the environmental load $K_{l}$ and $K_{2}$ are shown in Figure 2. It can be seen that environmental load $K_{l}$ of RAN 1 decreases significantly at simulation time 5 and the equilibrium is broken. At this time, if there is no adjustment, $N_{1}$ will decrease to 0 and $N_{2}$ will increase to the maximum value that the communication environment can support for network 2 according to the current competitive relationship between the two networks. On the other hand, if the adjustment exceeds a certain level, the equilibrium will also be broken due to over-increase of competition coefficient $\alpha_{21}$. In the situation, $N_{2}$ will decrease to 0 and $\mathrm{N}_{1}$ will increase to the maximum value that the communication environment can support for network 1.

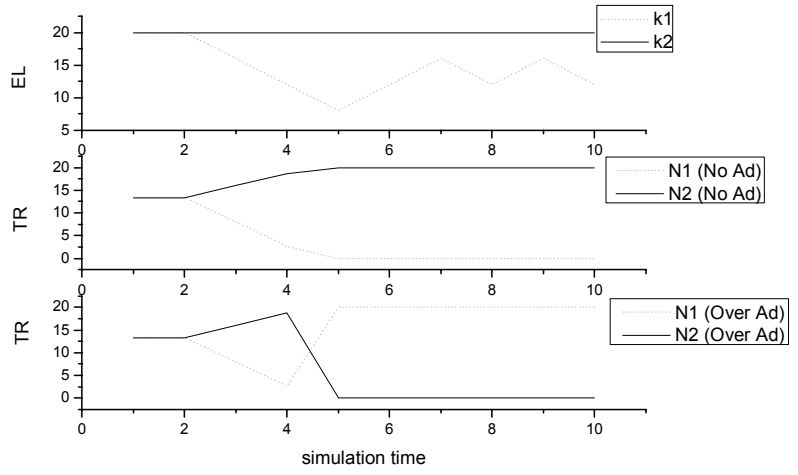

Fig. 2. Environmental load vs. traffic (no adjustment \& over adjustment)

\section{B. Adjustment Based on the L-V Model}

Under the same environmental load situation above, if the proposed adjustment algorithm is adopted, the adjustments of price and network support bandwidth as well as the change of the competition coefficients are shown in Figure 3.

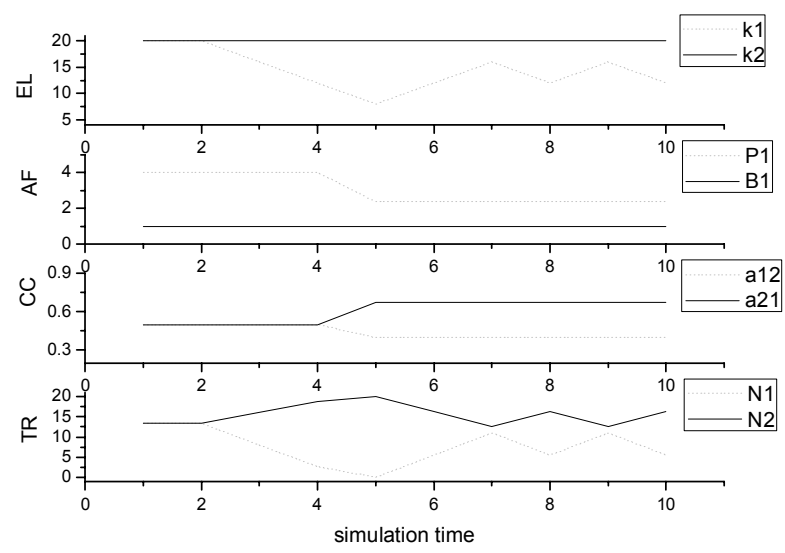

Fig. 3. Environmental load vs. traffic (adjustment based on L-V model)

The adjustment algorithm is triggered at simulation time 5. In this example, the two competitiive networks are restored to equilibrium by the decrease of $P_{l}$. It can also be seen that the decrease of $P_{1}$ will introduce changes on competition coefficients $\alpha_{12}$ and $\alpha_{21}$. The decrease of $\alpha_{12}$ ensures that the decrease of network 1 environment load $K_{l}$ will not cause network 1 to die out. Note that the adjustment of network support bandwidth is not necessary in this particular example.

Through adjustment using the above algorithm, two competitiive networks achieve another new equilibrium. At this time, although the decreasing of network 1 environmental load $K_{l}$ causes the decreasing of network 1 traffic $N_{l}$, the value will not go zero. As a result, these two networks can coexist and maintain traffic to a certain extent, as shown in Figure 3. 


\section{CONCLUSION}

In this article, heterogeneous wireless networks in JRRM are mapped into the L-V model and the relationship between the RANs are mapped into competitive relationship between different species. Accordingly, the JRRM adjustment algorithm based on the L-V model is proposed. Through this algorithm, distributed joint radio resource allocation will be obtained without impact on the coexistence $f$ the competitive networks. Simulation results show that the above adjustment algorithm can perform proper adjustment according to the current network situation. Compared with no adjustment and over adjustment, the proposed algorithm can keep the equilibrium of two RANs to ensure they maintain certain traffic load.

\section{REFERENCES}

[1] E. Gustafsson, A. Jonsson, , "Always best connected, " IEEE Wireless Communications, vol. 10, pp. $49-55,2003$

[2] J. Luo, R.Mukerjee, M. Dillinger, et al., "Radio resource management distribution in a Beyond 3G Multi-Radio Access Architecture Investigation of radio resource scheduling in WLANs coupled with $3 \mathrm{G}$ cellular network, " IEEE Communications Magazine, vol. 41 pp. $108-$ 115,2003

[3] A. Tolli, P. Hakalin, H. Holma, "Performance evaluation of common radio resource management (CRRM), " IEEE International Conference on Communications, vol. 5, pp. 3429 - 3433, Apr. 28, 2002

[4] Q. Song, A. Jamalipour, "Network selection in an integrated wireless LAN and UMTS environment using mathematical modeling and computing techniques, " IEEE Wireless Communications, Vol. 12, pp. 42 -48 , June 2005

[5] A. Furuskar, J. Zander, "Multiservice allocation for multiaccess wireless systems," IEEE Transactions on Wireless Communications, vol. 4, pp.174 - 184, Jan. 2005

[6] P. Demestichas, V. Stavroulaki, "Issues in introducing resource brokerage functionality in B3G, composite radio, environments, " IEEE Wireless Communications Magazine, vol.11 pp. 32-40, 2004

[7] P. Demestichas, V. Stavroulaki, L. Papadopoulou, A. Vasilakos, M. Theologou, "Service configuration and distribution in composite radio environments", IEEE Transactions on Systems, Man and Cybernetics Journal, vol. 33 pp. 69-81, 2004 